Case Report

\title{
Mucormycosis: A diagnostic and therapeutic challenge: A Case Report
}

\author{
D Wickramasinghe ${ }^{1}$, LD Liyanapathirana $^{1}$, NZ Miskin $^{1}$, LMS Sigera $^{2}$, PI Jayasekera ${ }^{2}$, \\ RLPR Liyanage ${ }^{3}$
}

Sri Lankan Journal of Infectious Diseases 2021 Vol.11(2):116-120

DOI: http://dx.doi.org/10.4038/sljid.v11i2.8348

\begin{abstract}
Mucormycosis is a fungal infection caused by omnipresent saprophytic fungi of the order Mucorales which occurs in humans and is life threatening. We present a patient in whom a prompt diagnosis followed by aggressive and appropriate treatment in a diabetic patient resulted in complete resolution of mucormycosis of the maxillary sinus.
\end{abstract}

Keywords: Mucormycosis, zygomycetes, amphotericin B

\section{Introduction}

Mucormycosis is a life threatening opportunistic fungal infection caused by saprophytic fungi of the order Mucorales. This infection usually occurs in post-transplant patients, patients on various immunosuppressive drugs, iron chelation therapy with desferrioxamine, patients with prolonged or profound neutropenia, uncontrolled diabetes mellitus, metabolic acidosis and malnutrition. High risk patient groups include hematopoietic stem cell transplantation recipients, solid organ transplant recipients and patients with haematological malignancies, Type I or II diabetes mellitus, burn patients and injection drug users. ${ }^{1}$ Mucormycosis has also been reported in immune competent population as well. ${ }^{2}$

We describe an atypical presentation of mucormycosis in the maxillary sinus of a patient with uncontrolled diabetes mellitus which was dealt with using an aggressive multimodal treatment approach. We emphasize the necessity of prompt diagnosis as well as rectifying underlying causes of immunodeficiency to offer the best chance of survival.

\footnotetext{
${ }^{1}$ District General Hospital, Hambantota Sri Lanka

${ }^{2}$ Medical Research Institute, Colombo 8, Sri Lanka

${ }^{3}$ National Dental Hospital, Colombo, Sri Lanka
}

Address for correspondence: Dr. Darshana Wickramasinghe, Consultant Microbiologist, DGH, Hambantota, Sri Lanka.. Telephone: +0094777627502/0094718669091 E-mail: dwickw2@yahoo.co.uk

Dttps://orcid.org/0000-0003-2140-068X

Received 14 January 2021 and revised version accepted 15 April 2021.

c) (7)

use, distribution, and reproduction in any medium, provided the original author and source are credited. 


\section{Case Report}

A 57-year-old male presented with pain in relation to a left molar tooth for 3 days. He also had difficulty in wearing his upper removable denture due to a projection on his left upper buccal sulcus for a week. He was a diagnosed type II diabetic patient who was not on regular medication for the past 8 years. Although he had gone to a dental surgeon for tooth extraction the previous day, the intra oral periapical radiograph taken showed no abnormality in the clinically sound tooth. Suspecting a sinus pathology, the dental surgeon referred the patient to an oral and maxillofacial (OMF) surgeon. As he complained of pain and numbness over his left side face, he was admitted to the hospital. The occipitomental view radiograph (Figure 1) showed a significant opacity in the left maxillary sinus. The CT scan appeared to be normal.

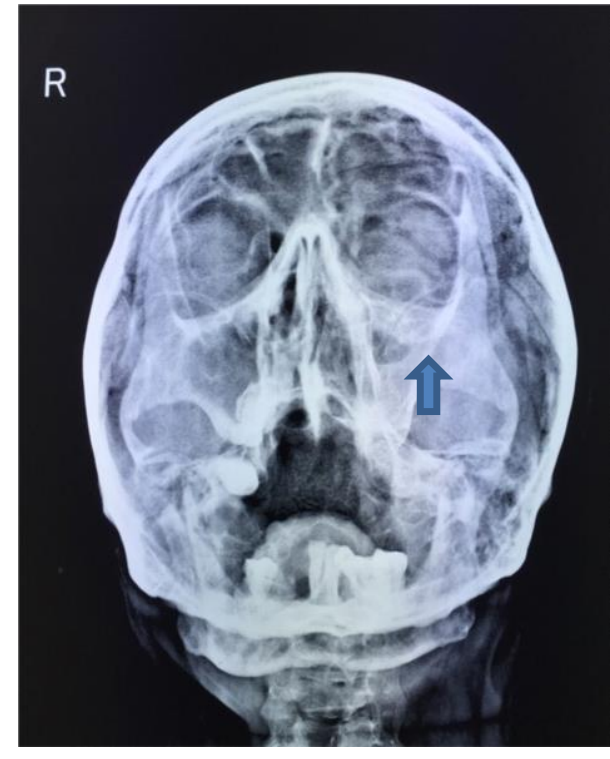

Figure 1: X-ray Occipitomental view showing opacity in the left maxillary sinus (arrow)
Extra oral examination did not show significant left sided facial swelling but mild tenderness over the left maxillary region was noted. On intra oral inspection, a mucosal swelling was noted on the left upper buccal sulcus with mild tenderness. The overlying mucosa was normal with no evidence of necrosis or discharge.

This patient was from a village background associated with farming. Although clinically the presentation was of a closed abscess, suspecting chronic sinusitis, left maxillary sinus exploration was carried out under local anaesthesia, after controlling his diabetes. Bone necrosis was noted extending from the left side palate to the pterygoid plate and zygomatic bone with minimal bleeding. Specimens were sent for histopathology and fungal studies to the Mycology reference laboratory.

Microscopic examination with $10 \% \mathrm{KOH}$ of the specimen showed wide, ribbon-like, non-septate hyaline hyphae. The rest of the sample was inoculated on Sabouraud dextrose agar with chloramphenicol for culture at $37{ }^{\circ} \mathrm{C}$ and $26{ }^{\circ} \mathrm{C}$.

The microbiology team advised IV amphotericin B (liposomal) as the direct smear was positive for fungal filaments. After education of the patient and his family, the nursing staff and the OMF team, IV liposomal amphotericin B was started. The trial dose (1mg IV infused over 1 hour) was successfully administered without any complications and continued with the therapeutic dose of $3 \mathrm{mg} / \mathrm{kg} / \mathrm{day}$.

After 5 days of incubation, a white filamentous growth extending up to the lid of the culture bottles was observed (Figure 2). Later it turned into a grey floccose colony. Wide, infrequently septate hyaline hyphae with pigmented rhizoids were observed in the lactophenol cotton blue mount of the growth (Figure 3). Sporangiophores were located on the nodes directly above the 
rhizoids and had sporangia on their tips. Sporangia were multispored, mostly globose and had hemispherical columellae.

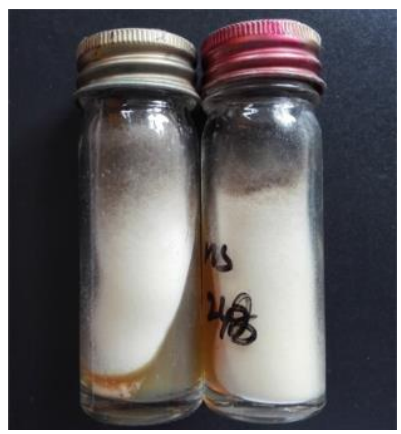

Figure 2.

White filamentous growth

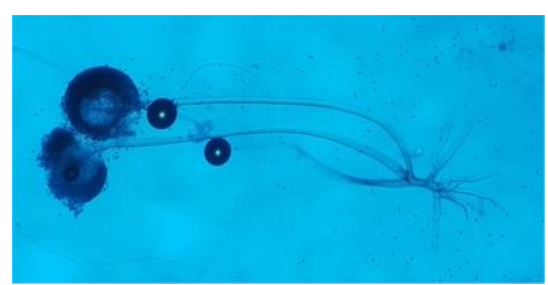

Figure 3. lactophenol cotton blue mount

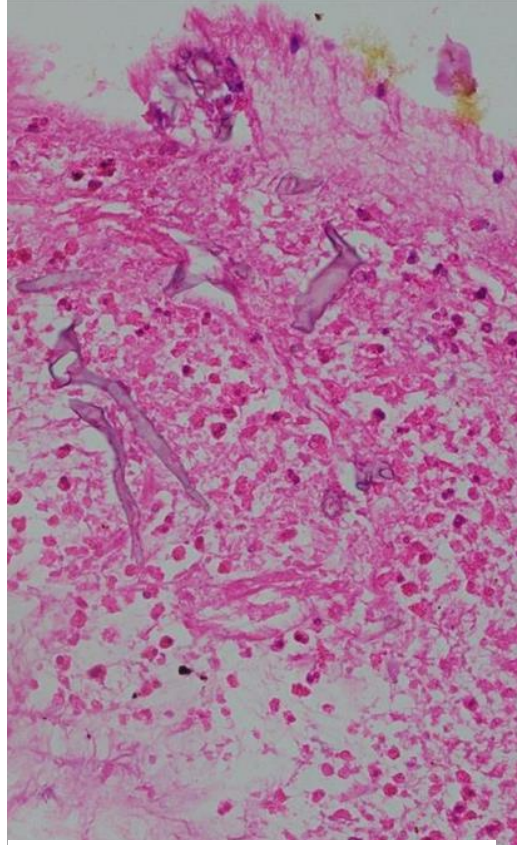

Figure 4. Wide, ribbon like, non- septate hyaline hyphae

The patient's glycaemic control was not satisfactory, and the condition was life threatening, as it had expanded beyond the site of origin. He required prolonged administration of IV amphotericin B which was challenging as it required slow intravenous administration. Amphotericin B was successfully administered for 17 days with no side effects followed by left maxillary sinus exploration and surgical debridement under general anaesthesia. During surgery, healthy bone tissue was noted. Specimens sent for fungal studies were positive for fungal filaments, but culture was negative. IV liposomal amphotericin B was continued for another 14 days, which made a total of 31 days. During this period his serum potassium levels were closely monitored, and potassium replaced appropriately. Close monitoring of renal and liver functions, full blood count, serum magnesium and calcium levels helped in tackling the drug adverse effects.

Extensive surgical debridement of the fungal material, quick and accurate diagnosis of the causative agent, achieving acceptable glycaemic control and accurate prompt commencement of systemic antifungal therapy with IV amphotericin B were the key aspects in management. After 31 days of systemic amphotericin B therapy our patient achieved a satisfactory clinical recovery and was discharged (Figure 5). On two weeks review he had an oroantral fistula at the surgical site and he is awaiting a follow up CT scan in one months' time.

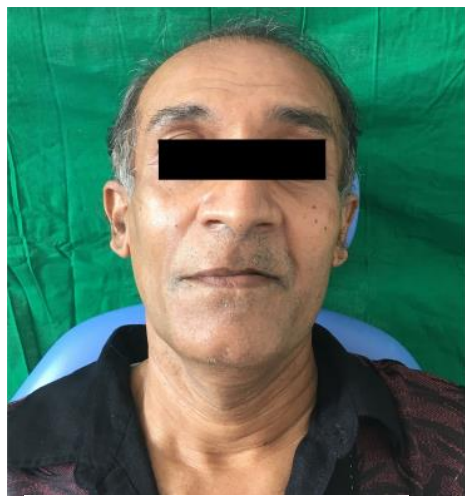

Figure 5.

Post-operative view 


\section{Discussion}

Earlier than bacteria, fungi were recognized as infectious agents of humans. ${ }^{4}$ Mucormycosis incorporates a large spectrum of infections caused by zygomycetes, a class of fungi that make branching ribbon like hyphae and reproduce sexually by formation of zygospores. These fungi may be found in fruits, soil, faeces, decaying matter and can also be cultured from the oral cavity, nasal passage and throat of healthy individuals. Infection can occur via inhalation, ingestion or traumatic inoculation. ${ }^{3}$ Rhinocerebral, pulmonary, gastrointestinal, cutaneous and disseminated mucormycosis are the main clinical presentations of the disease. ${ }^{3}$ Fungal invasion of the hard palate, paranasal sinuses, orbits and brain is the commonest form of rhinocerebral mucormycosis. ${ }^{3}$ Low host resistance trigger these avirulent fungi to become pathogenic. The normal response of the human body against infection is altered in diabetes mellitus and fungal cell proliferation is stimulated by hyperglycaemia. ${ }^{6}$

For sino orbital infections, the necrotic lesions and the surrounding infected tissue need early, aggressive and extensive surgical debridement which is of utmost importance as amphotericin B has poor penetration into those tissues. Management of mucormycosis largely depends on prompt diagnosis, reversal or minimizing underlying predisposing factors, repeated extensive infected tissue surgical debridement and prompt administration of correct systemic antifungal therapy for an adequate period of time.

A few antifungals show in vitro activity against mucormycetes. They include amphotericin B, posaconazole and isavuconazole. However, the latter two drugs are not widely available in Sri Lanka and are very expensive. Conventional azoles including fluconazole show no substantial activity against mucomycetes. ${ }^{5}$ The drug of choice for mucormycosis is amphotericin B. Side effects which need vigilant monitoring as well as an appropriate approach in educating the patient and associated medical team, complicates systemic amphotericin B therapy.

The optimal duration of systemic antifungal therapy for mucormycosis remains poorly defined. Treatment decisions are highly customized depending on the patients' clinical response and the rate of infection clearance. Until clinical and radiological abnormalities resolve, all mycological studies become negative and reversible underlying predisposing condition/s have abated, treatment should be continued. ${ }^{4}$

Late onset of associated symptoms such as pain and fever, hinder the early diagnosis, as profuse inflammation is not generated in fungal infections. Prevention always remains the gold standard. In immune compromised patients, maintaining immune balance plays a major role in preventing this fulminant infection.

\section{Conclusion}

Mucormycosis is a pernicious and aggressive fungal infection. In order to make a timely diagnosis a high level of clinical suspicion is essential. In this regard, as oral manifestations are the first to appear, especially in immune compromised patients, the OMF team plays an important role. To determine the diagnosis, histopathological and fungal studies are of great help. 
Comprehensive management includes precise identification of the fungal infection, correction of predisposing factors, adequate repeated extensive surgical debridement, appropriate prompt systemic antifungal therapy and methodical approach through patient education and increased awareness of medical teams associated with the treatment process to overcome anticipated complications of amphotericin B.

\section{Declarations}

Conflict of Interest statement - Authors declare no conflict of interest

Acknowledgments - We thank the patient and his family for consenting to the publication of the case details.

Funding source - None

Ethics statement and consent for publication - The patients' informed written consent was taken for publication.

Author contributions - DW was involved with clinical management, supervised laboratory procedures and manuscript writing; LDL was

involved with clinical management; NZM was involved with clinical management and wrote the manuscript; LMSS helped in manuscript writing and laboratory work;. PIJ carried out laboratory work and supervised manuscript writing; RLPRL was involved in laboratory work

\section{References}

1. Fungal infection Diagnosis Management, $4^{\text {th }}$ edition, Malcolm D. Richardson \& David W. Warnock, Wiley Blackwell publication 2012

2. Forgarty C, Regennitter F, Viozzi CF. Invasive fungal infection of the maxilla following dental extractions in a patient with chronic obstructive pulmonary disease. Journal of Canadian Dental Association. 2006; 72(2):149-152 PMID: 16545177

3. Ahamed SK, Thobaiti YA. Mucormycosis: A challenge for diagnosis and treatment -2 case reports and review of literature. Journal of Oral Health and Dental Management. 2014; 13(3):703-706 PMID: 25284541

4. Oswal NP, Gadre PK, Sathe P, Gadre KS. Mucormycosis of mandible with unfavorable outcome. Case Reports in Dentistry.2012; Article Id 257940 doi: https://doi.org/10.1155/2012/257940

5. Pilmis B, Alanio A, Lortholary O,Lanternier F. Recent advances in the understanding and management of mucormycosis, 2018; F1000 Faculty Rev-1429. doi: 10.12688/f1000research.15081.1.

6. Afroze SN, Korlepara R, Rao GV, Madala J. Mucormycosis in a Diabetic Patient: A Case Report with Insight into Its Pathophysiology. Contemporary Clinical Dentistry.2017; 8(4):662-666. doi:10.4103/ccd.ccd_558_17 